aims and shielded from the vicissitudes of a competitive commercial environment, would be a substantial and practicable alternative.

Service benefits of integration include one stop care for patients and continuous professional collaboration in establishing and maintaining practice formularies, using drugs and auditing their costs, purchasing, managing budgets, overseeing complex and costly therapeutic regimens, conducting postmarketing surveillance, and reporting adverse reactions. In a recent book, Marinker and Reilly argued that "Primary care pharmacists, based in practices, could become responsible for the pharmaceutical care of the practice population. They would also effect liaison with community and hospital pharmacists, would undertake domiciliary visits where necessary and would certainly emerge as key players in the primary health care team of the future."

As about two thirds of the total drug bill arises from repeat prescriptions, urgent professional collaboration is needed to address this issue. Integration of the professions is the perfect vehicle to deliver rapid and sustained improvements in all aspects of repeat prescribing. Currently, people who ask a pharmacist for advice are often referred for extra consultations with their general practitioner, with consequent delays. This potentially useful source of health care could be made much more effective with a unified team working on a single site.

Integration could yield annual savings of $£ 1 \mathrm{bn} .{ }^{6}$ Following the introduction of the option for NHS patients (those not exempt from prescription charges) of receiving a private prescription when it would be to their financial advantage, further savings would arise. The number of prescriptions whose value is less than the prescription charge would increase as salaried primary care pharmacists would not need to add a dispensing fee. Ending price maintenance on pharmaceuticals, certainly those whose patent has expired, would yield yet more savings.

Currently, pharmacists and dispensing doctors are the sole beneficiaries of their purchasing acumen. Direct billing of family health services authorities by suppliers could lead to additional savings for the NHS. Concentrating dispensing transactions in fewer locations (only in general practices) and making those pharmacists who supervise NHS dispensing salaried employees would facilitate financial control and would minimise the incentive to fraudulent practice, identified in a recent report by the Audit Commission. ${ }^{7}$

Such arrangements could undergo the necessary piloting almost immediately in existing dispensing practices and others with adequate accommodation. Parliament would have to change the law relating to general practice and pharmacy for the benefits to be realised by the entire population.

With only dispensing based in NHS primary care financed from taxation, where would this leave retail pharmacy? The prosperity, ubiquity, and quality of retail pharmacy could be assured by several changes. Firstly, a monopoly on the sale of all over the counter pharmaceuticals could replace the existing monopoly on "pharmacy only" items, ensuring a measure of professional supervision of sales and facilitating a concentration on the commercial aspects of promoting health and hygiene and managing minor illness. More products could be transferred from prescription only to pharmacy only status. Secondly, as a guard against commercial considerations overriding therapeutic validity, records that could be audited should be considered for some of the more active products sold direct to the public. Thirdly, the removal of price maintenance would promote competitive pricing. Finally, as retail pharmacy would become free of any constraint on the location of new premises, areas previously unable to sustain a pharmacy could then benefit.

STEVEN FORD General practitioner

Haydon Bridge,

Northumberland NE47 6HJ

University of Newcastle upon Tyne, Newcastle upon Tyne NE2 4HH

Senior lecturer in primary health care

\footnotetext{
1 Pearson P, Jones K. The primary health care non-team? BMF 1994;309:1387-8.

2 Brown P. Are pharmacists necessary? Scrip Magazine 1994 May:3-4.

3 Brown P. Pharmacy's rocky road. Pharmaceutical Foumal 1994;252:802-3.

4 Clucas K. Pharmacy. A report to the Nuffield Foundation. London: Nuffield Foundation, 1986.

5 Marinker M, Reilly $P$. Judging rational prescribing. In: Marinker $M$, ed. Controversies in health care policies, challenges to practice. London: BMJ Publishing Group, 1994:89-110.

6 Ford S. Community pharmacy. Br 7 Gen Pract 1994;388:534-5.

7 Audit Commission. Protecting the public purse 2-ensuring probity in the NHS. London: HMSO, Audit Commiss
1994:63.
}

\title{
Chemoprophylaxis in tuberculosis and HIV infection
}

\author{
Is it feasible in developing countries?
}

Should chemoprophylaxis with a course of antituberculous drugs be given to people infected with Mycobacterium tuberculosis and HIV to prevent the reactivation of latent tuberculosis? The decision depends on three factors: the efficacy of the treatment, its cost effectiveness, and whether it is financially and organisationally feasible.

The World Health Organisation estimates that 1700 million people are infected with $M$ tuberculosis-one third of the world's population. Symptomatic tuberculosis is becoming an increasing problem owing to population growth, poverty, multidrug resistance, and HIV infection. The risk of active tuberculosis in dually infected people is 3-8\% a year, with a lifetime risk of about $50 \%$ or more. ${ }^{1}$ The WHO has calculated that the global incidence in 1990 of about 7.5 million cases of tuberculosis will increase to about 12 million by 2005 (M C Raviglione, personal communication). Already, worldwide, tuberculosis is the most common cause of death due to a single infectious agent.
Chemoprophylaxis with isoniazid has an efficacy of 25-92\% in various risk groups not infected with HIV. ${ }^{2}$ Some data are available in people infected with HIV. In a controlled trial in Zambia patients with HIV infection and with Walter Reed stages III and IV disease who were given a $B$ vitamin placebo had an incidence of tuberculosis of 11.2 per 100 person years, which was four times the rate in those receiving daily isoniazid (D Wahhawan et al, eighth international conference on AIDS and third world congress on sexually transmitted diseases, Amsterdam, 1992).

In Haiti a 12 month course of prophylaxis with isoniazid reduced the incidence of tuberculosis from 10.0 to 1.7 per 100 person years and also delayed the progression of symptomless HIV infection to active disease and death by an average of $9 \cdot 7$ and 5.3 months respectively. ${ }^{3}$ In addition to preventing reactivation of the tuberculosis chemoprophylaxis may also prevent the activation of HIV infected CD4 lymphocytes by $M$ tuberculosis and thus the progression from HIV infection to 
clinical AIDS. ${ }^{4}$ Indeed, it may be the only hope of prolonging life in HIV positive people in developing countries.

Numerous clinical trials in populations not infected with HIV have shown no evidence of the development of drug resistant disease during chemoprophylaxis. ${ }^{2}$ Treatment does, however, selectively increase the proportion of resistant strains, ${ }^{5}$ and hence widespread chemoprophylaxis with isoniazid might considerably increase the level of resistance in the community (D A Mitchison, personal communication). Moreover, in people with HIV infection, excluding the possibility of active tuberculosis is more difficult: screening procedures are essential to reduce the risk of starting chemoprophylaxis in people with active disease. Shorter multidrug regimens with rifampicin are being studied; these are likely to reduce the risk of undiagnosed active disease and improve efficacy and compliance but increase the cost.

The degree of compliance (and the risk of drug resistance) is likely to vary according to the site at which HIV infection is diagnosed. At a voluntary testing centre in Kampala, Uganda, results of preliminary research showed low recruitment $(28 \%)$ of HIV positive people to chemoprophylaxis because of procedural problems but a $63 \%$ compliance rate among those who started the six month course of isoniazid. ${ }^{6}$ Further research is needed to confirm the efficacy of various drug regimens; to determine at what stage in the course of HIV infection chemoprophylaxis is most appropriate; and to determine the optimal frequency of taking treatment, the duration of treatment, and how best to monitor it. We also need to know the risk of reinfection and hence whether continued prophylaxis is necessary.

Although the cost effectiveness of chemoprophylaxis is not yet established, some indication of the costs is available. The benefits can be calculated from the costs of treatment of active disease, increased life expectancy, and prevention of transmission. In the Ugandan study the incremental cost of adding the drugs and screening required for preventive treatment was estimated to be $\$ 11.93$ over and above the existing $\$ 18.25$ for HIV testing and counselling at the voluntary testing centre and $\$ 7.89$ for follow up counselling and social support. This compares favourably with the overall cost of $\$ 38.78$ for the standard 12 month regimen for symptomatic tuberculosis in Uganda ( $\mathrm{T}$ Aisu et al, ninth international conference on AIDS, Berlin, 6-11 June 1993).

\section{Costs seem low}

Moreover, the treatment of patients who are positive for tuberculosis on smear testing but are not HIV positive is known to be one of the cheapest health interventions available in developing countries, at a cost of only \$1-4 per year of life saved compared with $\$ 5-10$ for childhood immunisation. ${ }^{6}$ The direct benefits of treating tuberculosis in people with HIV infection are likely to be considerably less owing to their reduced life expectancy, but treatment will reduce transmission to others. There may also be benefits in preventing AIDS through the changed sexual practices and use of condoms resulting from the counselling that is part of the assessment for chemoprophylaxis.

Even if chemoprophylaxis is found to be cost effective, however, we still have to ask if it is financially and organisationally feasible. Might it divert scarce resources from the treatment of patients positive for tuberculosis on sputum testing? Chemoprophylaxis for the whole population of people with tuberculosis and HIV infection would be an immense task. In Uganda, for example, this would require the identification and subsequent treatment of more than one million HIV positive people in an adult population of 16 million. ${ }^{7}$ The alternative- providing treatment to more than a third this number of people as they develop active tuberculosis-is also daunting. Both tuberculosis programmes and general health services are severely underfunded, and they may be unable to pay for preventive treatment, however cost effective it might be in reducing future caseloads. Programmes to control AIDS may be better funded, but they face the inevitable and huge cost of providing care for people with AIDS and so may not be able to pay for chemoprophylaxis. ${ }^{8}$

A joint statement by the WHO's tuberculosis programme and global programme on AIDS and the International Union Against Tuberculosis and Lung Disease has re-emphasised that the primary objective of a tuberculosis programme is to interrupt the transmission of tuberculosis by the curative treatment of infectious tuberculosis. ${ }^{-}$That implies that chemoprophylaxis should be considered only in areas where tuberculosis programmes are already successfully achieving the WHO's targets of detecting $70 \%$ and treating $85 \%$ of patients found to be positive on smear testing - and where voluntary testing and counselling for HIV infection are also available. These conditions are not met in most developing countries.

After years of neglect major inputs by the WHO, the World Bank, the Overseas Development Agency, and other agencies are helping national tuberculosis programmes to achieve the targets. Meanwhile, a limited approach may be possible, targeted at particular opportunities for screening in areas of high prevalence and groups with high risk behaviour. The financial and organisational feasibility-especially the follow up procedures required for high rates of compliance-may be greatest in occupational groups such as mine and factory workers, the military, and the police. Chemoprophylaxis is also likely to be more feasible in middle income Asian and Latin American countries. The ability and willingness of patients to meet all or part of the cost must also be studied.

If the current studies confirm that chemoprophylaxis is effective in developing countries it will be reasonable for clinicians to provide isoniazid for six to 12 months for individuals - that is, people who for any reason have been tested and found to be HIV positive and in whom active tuberculosis has been excluded. Decisions to start chemoprophylaxis control programmes will depend on the demonstration of cost effectiveness as well as the provision of adequate financial resources. These require operations research, which should be given high priority.

JOHN WALLEY
Senior lecturer in international public health

International Division,

Nuffield Institute for Health,

Leeds LS2 9PL

JOHN PORTER Senior lecturer

Department of Clinical Sciences,

London School of Hygiene and Tropical Medicine, London WC1E 7HT

1 Selwyn PA, Hartel D, Lewis VA, Schoenbaum E, Vermund SH, Walker T, et al. A prospective study of the risk of tuberculosis among intravenous drug users with human immunodeficiency virus infection. N Engl F Med 1989;320:545-50.

2 O'Brien RL. Preventive therapy for tuberculosis. In: Porter J, McAdam K, eds. Tuberculosis-back to the future. London: Wiley, 1933.

3 Pape WP, Simone SJ, Ho JL, Hafner A, Johnson WD. Effect of isoniazid prophylaxis on incidence of active tuberculosis and progression of HIV disease. Lancet 1993;342:268-72.

4 Edwards D, Kirkpatrick C. The immunology of mycobacterial diseases. Am Rev Respir Dis 1986;134:1062-71.

5 Ferebee SH. Controlled chemoprophylaxis trials in tuberculosis: a general review. Advances in Tuberculosis Research 1970;17:29-105.

6 Jamison DT, Mosley WH, eds. Evolving health sector priorities in developing countries. Washington, DC: World Bank, 1991.

7 Goodgame RW. AIDS in Uganda: clinical and social features. N Engl f Med 1990;323:383-7.

8 Schopper D, Walley J. Care for AIDS patients in developing countries: a review. AIDS Care 1992;4:89-102.

9 World Health Organisation. Tuberculosis preventive therapy in HIV-infected individuals: a joint statement of the WHO tuberculosis programme and the global programme on AIDS, and the International Union Against Tuberculosis and Lung Disease (IUATLD). Wkly Epidemiol Rec 1993;49:361-4. 